\section{P-114 MORTALITY AUDIT OF CARE HOME PATIENTS KNOWN TO THE COMMUNITY PALLIATIVE CARE TEAM}

Maxine Concannon, Michelle Tombs, Claire Capewell. St Catherine's Hospice, Preston, UK

10.1136/bmjspcare-2019-HUKNC.137

Background The percentage of patients dying in care homes is steadily increasing, with $70 \%$ of permanent residents dying in their care home (Public Health England, 2017). Therefore, it is important to understand what is happening to the local population of care home residents at the end of life and their preferences for care.

Aims To assess the impact of involvement of Specialist Palliative Care, for care home patients, on deaths in usual place of residence, hospital admissions in last 90 days of life as well as length of stay in hospital in the six months prior to death. To assess the impact of advance care planning.

Methods A retrospective review of patients who were resident in care homes was undertaken. These patients were known to the Community Palliative Care Team and died between 16 September and 16 December 2018. The electronic patient records were used to gather the data, alongside requesting information from Business Intelligence at the local hospital Trust to obtain information regarding hospital admissions.

Results 74\% of patients achieved their Preferred Place of Death (PPD) in this audit. 2/3 of those not achieving PPD had requested hospice transfer. $89 \%$ died in the care home and only $4 \%$ died in hospital. However, $41 \%$ of patients had three or more admissions to hospital within the last six months of life. 93\% had documented evidence of having a DNACPR (Do Not Attempt Cardio-pulmonary Resuscitation) form in place and there was evidence of advance care planning for $85 \%$ of patients.

Conclusions Further exploration is required around the reasons these patients are being admitted to hospital. Our action plan includes signposting care homes to available education, sharing learning with generalist colleagues and encouraging After Death Analysis at GSF meetings.

\section{Patient Care}

\section{P-115 INTRODUCTION OF NEWS2 IN A HOSPICE SETTING}

Helen Ireland, Kate Davies, Jane Donohue, Bethany Wright, Gaye Senior-Smith. St Peter's Hospice, Bristol, UK

\subsection{6/bmjspcare-2019-HUKNC.138}

Background Following a clinical incident there was a review of the current system for recording and reporting patient observations. This involved an initial audit of current practice and scoping of regional and national practice.

Aims The project aim was to provide all clinical staff with a tool to identify critically unwell patients and respond safely and appropriately.

Methods The project team looked at the local hospitals NEWS (National Early Warning Score) charts and embarked on designing a bespoke chart for the hospice setting. Following several rounds of consultation and different iterations it happened that the NEWS 2 (Royal College of Physicians. National Early Warning Score (NEWS) 2, 2017) tool was released nationally. This led to further review and highlighted our error in trying to adapt a nationally recognised tool which was enabling common language, transferability and standardisation across different settings.

Results Following cascade training to all clinical staff, NEWS 2 is now embedded in use in the Inpatient Unit. Staff have said it 'highlights when we should respond to triggers' and it 'helps decision making overnight and when the medical on call needs to be contacted'. Weekly snapshot audits have also shown examples of clearer documentation regarding planned frequency of observation and clinical response and allowed assessment and teaching at the point of care. A more formal audit is being undertaken in June 2019 and the results of this will also be reported.

Conclusion Despite some initial resistance to change we have shown that it has been possible and acceptable to introduce the use of NEWS 2 in the hospice inpatient setting. Following this it is now being rolled out to other departments including Day Services and the Clinical Nurse Specialist Team.

\section{P-116 WHAT ARE HOSPICE STAFF PERCEPTIONS OF CARING FOR PATIENTS WITH APHASIA?}

${ }^{1}$ Louise Shelley, ${ }^{2}$ Janet Holt. ' Leeds Teaching Hospitals NHS Trust, Leeds, UK; ${ }^{2}$ University of Leeds, Leeds, UK

\subsection{6/bmispcare-2019-HUKNC.139}

Background Aphasia is an acquired disorder of language and can be caused by conditions including stroke, brain tumour, head injury and dementia (Royal College of Speech \& Language Therapists, 2019). Due to the nature of these conditions, it is likely that people with aphasia will access palliative care (Hawksley, Ludlow, Buttimer \& Bloch, 2017).

Speech and language therapists (SLTs) are well placed as 'communication experts' to support patients with aphasia to communicate. Although the role of SLTs in palliative care is well documented (Chahda, Mathisen \& Carey, 2017) the literature suggests that SLTs are not usually integrated within a hospice MDT (Pollens, 2004).

Aim To identify views of hospice staff on their interactions with patients with aphasia.

Research questions

- Do staff have an understanding of SLT and do they feel SLTs have a role in palliative care?

- Do staff feel confident in interacting with patients with aphasia and/or do they feel that service improvements need to be made to improve these interactions?

Methods A qualitative descriptive exploratory design (Sandelowski, 2000). Four doctors and four nurses working in hospices in Yorkshire were interviewed using a semi-structured interview guide. Hospices which identified that they commonly care for patients with aphasia were included. A purposive sampling strategy was adopted. Data were analysed using thematic analysis (Tesch, 1990).

Results The following themes emerged:

- Knowledge of and attitudes towards speech and language therapy;

- Knowledge and awareness of supporting people with communication impairments;

- Palliative care in the hospice;

- Using family to support;

- Directions for change. 